\title{
DETERMINACIÓN DEL CALOR ESPECÍFICO DE LOS ACEITES TÉRMICO Y DE SOYA Y SU EFECTO EN LA EFICIENCIA ENERGÉTICA DE LA CALDERA DE FREIDORA DE PAPAS.
}

\section{DETERMINATION OF THE SPECIFIC HEAT OF THE THERMIC OIL AND SOYA BEAN OIL AND HIS EFFECT IN THE ENERGETIC EFFICIENCY OF THE BOILER OF POTATOES FRYER.}

\author{
PhD. Francisco García Reina ${ }^{1}$, MSc. Frank Cordero Rodríguez ${ }^{2}$, Lic. Roberto \\ Ramírez Zamora $^{\mathbf{1}}$, MSc. Francisco Hernández Rodríguez ${ }^{\mathbf{1}}$. \\ * Universidad de Ciego de Ávila (UNICA), Facultad de Ciencias Técnicas, Grupo de \\ Investigación en Energía. \\ Carretera de Morón Km 9, Ciego de Ávila, Cuba. \\ Teléfono: 53-33-225702, ext. 1060. \\ E-mail: pancho30011918@gmail.com. \\ **UEB Industrial Ceballos, Combinado Cítrico. \\ Carretera de Carrtera de Ceballos Km 8.Ciego de Ávila, Cuba.
}

\begin{abstract}
Resumen: En la actualidad se emplean cada vez más en la cocción de alimentos las calderas que usan aceites térmicos debido a su bajo calor específico comparado con el agua, por lo que estas calderas son más eficientes que las de vapor. Sin embargo para su correcto empleo es necesario medir con frecuencia el calor específico de los aceites, tanto el del térmico como el de soya, usados en esta instalación, para el control de la eficiencia de transferencia de calor en el intercambiador de la caldera. Usando un calorímetro de alta precisión del laboratorio de Eficiencia Energética de la UNICA se midieron los aceites empleados y se obtuvo para el térmico un calor específico de $1,425 \mathrm{~kJ} / \mathrm{kgK}$, mientras que el de soya es de $1,634 \mathrm{~kJ} / \mathrm{kgK}$, antes se usarse en la caldera. Las mediciones con muestras de estos aceites en función del tiempo de uso arrojó que el aceite térmico empleado es muy estable y puede usarse hasta un período de 6 meses sin pérdidas apreciables, no siendo así con el de soya, que al estar en contacto directo con las papas, se degrada muy rápido y calor específico supera $\operatorname{los} 2 \mathrm{~kJ} / \mathrm{kgK}$ a las 72 horas de uso, por lo que se recomienda sustuirlo antes de las 48 horas.
\end{abstract}

Palabras clave: calor específico, aceite térmico, aceite de soya, calderas.

\begin{abstract}
At this time, they are used every time in the cooking of foods the boilers that use thermal oils due to their low specific heat compared with the water, for what these boilers are more efficient than those of water. However, for their correct employment it is necessary to frequently measure the specific heat of the oils, so much which of the thermal one as that of soya, used in this installation, for the control of the efficiency of heat transfer in the heat interchanger of the boiler. Using a high precision calorimeter of the laboratory of Energy Efficiency of the UNICA one the used oils was measured and it was obtained for the thermal one a specific heat of $1,425 \mathrm{~kJ} / \mathrm{kgK}$, while that of soya is of $1,634 \mathrm{~kJ} / \mathrm{kgK}$, before you to be used in the boiler. The measurement with samples of these oils in function of the time of use threw that the oil thermal employee is very stable and it
\end{abstract}


can be used until a period of 6 months without appreciable losses, not being this way with that of soya that when being in direct contact with the potatoes, it demeans very quick and specific heat overcomes the $2 \mathrm{~kJ} / \mathrm{kgK}$ at the 72 hours of use, for what substitution is recommended before the 48 hours.

Keywords: specific heat, thermic oil, soy bean oil, boilers.

\section{Introducción:}

En la actualidad se emplean cada vez más en la cocción de alimentos las calderas que usan aceites térmicos debido a su bajo calor específico comparado con el agua, por lo que estas calderas son más eficientes que las de vapor. La importancia de conocer la eficiencia térmica con que operan la caldera de calentamiento de aceite térmico, radica en evaluar el grado de aprovechamiento de la energía del combustible. Además, se pueden cuantificar las cantidades de calor que ingresan y egresan de una caldera (Golato, 2008). (Marcano, 2012).

En las caldera de calentamiento y distribución de aceite térmico, cuya función es la transferencia de calor al aceite comestible mediante un intercambiador de carcaza-tubo en el proceso de fritado de la papa, es una novedad determinar la eficiencia de la caldera a partir de las mediciones de los calores específicos de ambos aceites, ya que es la magnitud física principal que gobierna el intercambio de calor entre ellos (Avella, 2012). Luego, para su correcto empleo es necesario medir con frecuencia el calor específico de los aceites, tanto el térmico como el de soya usados en esta instalación, para el control de la eficiencia de transferencia de calor en el intercambiador de calor de la caldera. En este trabajo se presenta un método para evaluar la eficiencia en la transferencia de calor entre ambos aceites, a partir de mediciones precisas del calor específico de ambos y de su evolución con el tiempo de uso.

Materiales y métodos.

En la UEB Industrial Ceballos cuenta con una línea procesadora de papas pre fritas única de su tipo en Cuba, en la cual se cuenta con una caldera de calentamiento del aceite térmico y un intercambiador de calor para la transferencia al aceite de soya, el cual es el que fríe las papas. Teniendo en cuenta que este es uno de los productos de mayor consumo por el hombre en el país. En muchas latitudes, La productividad de dicha línea es continua. Su diagrama de flujo se presenta en la figura $1 \mathrm{y}$ en la figura 2 imágenes de la caldera.

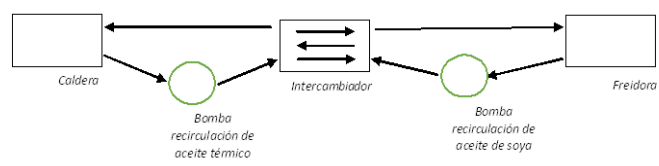

Figura 1: Diagrama de flujo de freidora de papas.
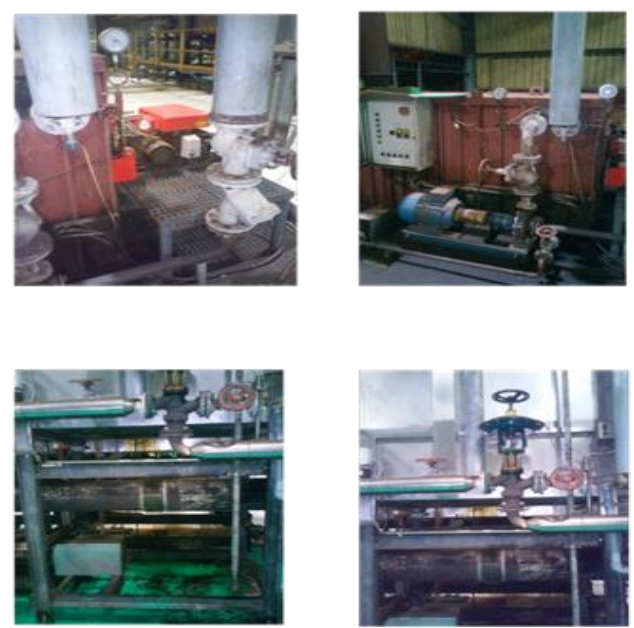

Figura 2: Imagen de la caldera de la freidora de papas

El aceite térmico usado es: CASTROL PERFECTO HT5, cuyos datos técnicos son:

- La densidad $15^{\circ} \mathrm{C}$ g / ml ASTM D4052 0.868

- La viscosidad $40^{\circ} \mathrm{C}$ cSt ASTM D445 30.5

- La viscosidad $100{ }^{\circ} \mathrm{C}$ cSt ASTM D445 5.28

- Viscosidad Índex ASTM D2270 106

- El derrame Point C ASTM D97 - 9

- El punto de inflamación, $\mathrm{PMC}^{\circ} \mathrm{C}$ ASTM D93 210 
- $\quad$ El punto de inflamación, $\mathrm{COC}^{\circ} \mathrm{C}$ ASTM D92 220

El aceite de soya es suministrado por la planta procesadora de Santiago de Cuba, certificado como apto para el consumo humano, pero sin datos técnicos.

Para la determinación de los calores específicos de ambos aceites se usó el calorímetro de alta precisión HPCI-231 de la firma Jianxe de la RPCh, con sensores de temperatura acoplados por interface a una PC. La imagen del sistema montado y su esquema se presentan en la figura 3 .
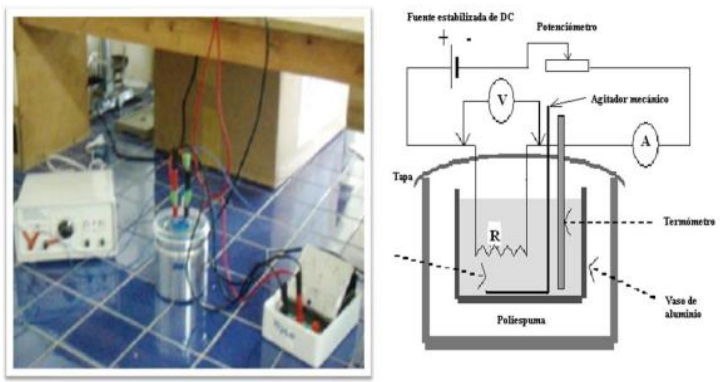

Figura 3: Calorímetro.

El fundamento de la medición es el siguiente: La potencia liberada en forma de calor por un conductor de resistencia $\mathrm{R}$, por el que circula una corriente de intensidad I, viene dada por la ley de Joule-Lenz:

$$
P=I V
$$

$\mathrm{O}$ teniendo en cuenta que esta potencia se libera en forma de calor:

$$
\frac{d Q}{d t}=I V
$$

Que en condiciones estacionarias $(I=$ cte. en el tiempo)

$$
\frac{Q}{t}=I V
$$

ó

$$
Q=I V t
$$

Usando la ley de $\mathrm{Ohm}, V=I R$ tiene las formas:

$$
Q=I^{2} R t=\frac{V^{2}}{R} t
$$

En el montaje empleado en esta medición, el calor desprendido por el conductor de resistencia $R$, con una intensidad de corriente $I$ y a una diferencia de potencial $V$, calienta una masa de líquido encerrada en un calorímetro. Midiendo la temperatura que alcanza esta masa de agua junto con el calorímetro al cabo de un tiempo $t$ se puede determinar el calor absorbido, el cual fue cedido por el conductor con corriente.

Como es sabido, el calor necesario para elevar la temperatura desde $T_{1}$ hasta $T_{2}$ de un cuerpo de masa $m$ viene dada por la ecuación:

$$
Q=m c\left(T_{2}-T_{1}\right)
$$

Donde $c$ es el calor específico de la sustancia del cuerpo, cuyo significado es la cantidad de calor necesario para elevar la temperatura $1^{\circ} \mathrm{C}$ de $1 \mathrm{~kg}$ de esta sustancia. Lógicamente si el cuerpo se enfría desde una temperatura $T_{2}$ hasta una temperatura $T_{l}$ el calor cedido por el cuerpo es el mismo que el absorbido según (4), pero con el signo cambiado. Es decir, el calor absorbido es +, mientras que el cedido es -.

Parta las mediciones termodinámicas se emplea frecuentemente al agua, ya que sus propiedades termodinámicas son muy conocidas. Sin embargo, está claro que el recipiente que contiene al agua junto con los equipos de medición y auxiliares (a este sistema se le llama calorímetro), que están en contacto con el agua, participan en el intercambio calorífico y hay que tenerlos en cuenta en la medición. Cada calorímetro tiene sus propias características térmicas que hay que determinar previo al experimento. En este caso se determina la capacidad calorífica $C$ del calorímetro, que es el calor necesario para elevar su temperatura $1^{\circ} \mathrm{C}$ y el calor absorbido o cedido por este viene dado por:

$$
Q_{\text {cal }}=C \Delta T
$$

Luego el balance calórico entre el conductor con corriente, el agua y el calorímetro será:

$$
\begin{array}{r}
Q=Q_{H_{2} O}+Q_{c a l} \\
Q=m c\left(T_{2}-T_{1}\right)+c \Delta T
\end{array}
$$

Donde en esta ecuación $Q$ es el calor suministrado por la resistencia con corriente, dado en la expresión (2). Con esta expresión (6) se determina la capacidad calorífica $C$ del calorímetro usando, para lo cual se usa el valor conocido del calor específico del agua $\left(c_{H 2 O}=4,1784 \mathrm{~kJ} / \mathrm{KgK}\right.$ a 30 $\left.{ }^{\circ} \mathrm{C}\right)$. Con este valor $C$ y la expresión (6) se determinan entonces el calor específico de los aceites estudiados, usando el mismo procedimiento que en el agua. 


\section{Resultados y su discusión:}

En la figura 4 se presenta el gráfico del calentamiento del agua pura en función del tiempo:

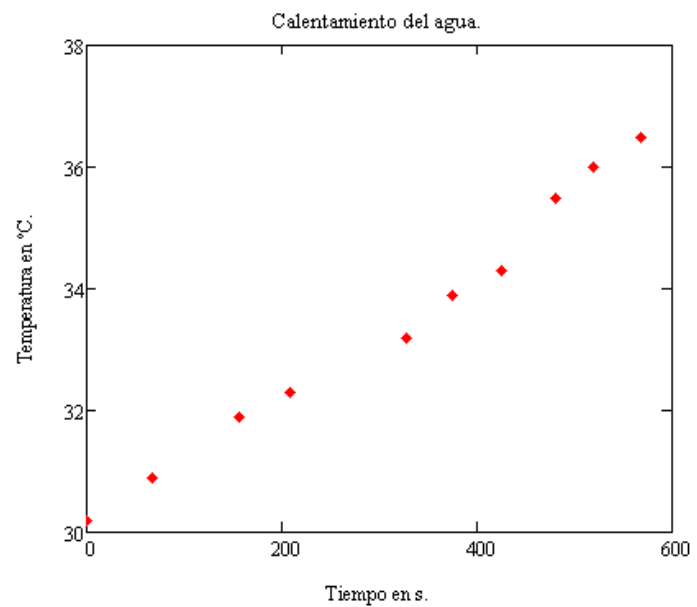

Figura 4: Calentamiento del agua pura.

Con estos valores y usando la expresión (6) se determina la capacidad calorífica $C$ del calorímetro, para la que se obtuvo $C=135,7 \pm 0,1$ $\mathrm{J} / \mathrm{K}$.

En la figura 5 se presenta el gráfico del calentamiento del aceite térmico en función del tiempo:

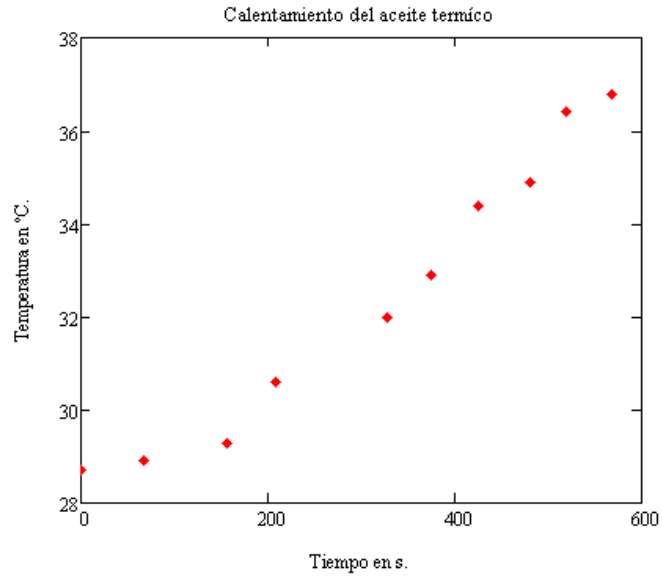

Figura 5: Calentamiento del aceite térmico.

Con estos valores y usando la expresión (6) se determina la calor específico del aceite térmico sin usar, para el que se obtuvo $c_{t}=1.853 \pm 0,005$ $\mathrm{kJ} / \mathrm{kgK}$, nótese que es casi 4 veces menor que el del agua y por esto su eficacia en el intercambio térmico, es decir necesita casi 4 veces menos calor para elevar su temperatura en $1 \mathrm{~K}$.
En la figura 6 se presenta el gráfico del calentamiento del aceite térmico en función del tiempo:

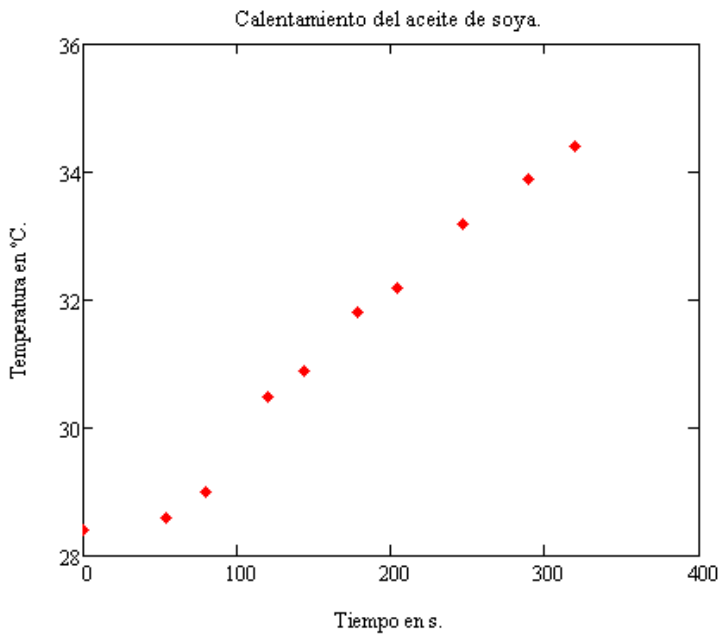

Figura 6: Calentamiento del aceite de soya.

Con estos valores y usando la expresión (6) se determina la calor específico del aceite de soya sin usar, para el que se obtuvo $c_{s}=2.303 \pm 0,005$ $\mathrm{kJ} / \mathrm{kgK}$, mayor que el térmico, pero mucho menor que el del agua, como se en la figura 7 con el ajuste a MC de las tres velocidades de calentamiento.

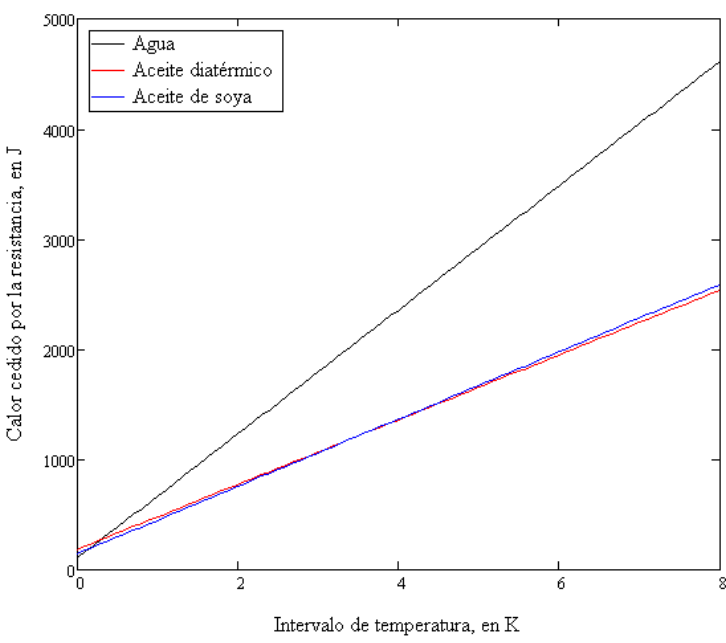

Figura 7: Cantidad de calor necesaria para subir la temperatura en $1^{\circ} \mathrm{C}$.

El calor específico del aceite térmico se incrementó en menos de un $2 \%$ después de 6 meses de uso, por lo que puede usarse durante mucho tiempo sin necesidad de cambio. Sin embargo, en el caso del aceite de soya, al estar en contacto con las papas en el proceso de fritado, se incrementa rápidamente llegando a ser casi el doble en 72 horas de uso, por lo que de igual manera se incrementa notablemente 
la cantidad de calor que hay que suministrarle a este aceite para elevar su temperatura, lo que provoca una caída de eficiencia energética de la caldera en la misma proporción. Por esta razón debe de ser sustituido después de 48 horas de uso, incluso antes que las 72 horas recomendadas para no afectar la salud del consumidor del producto (Fazina, 2014) (Nwafor, 2003).

\section{Conclusiones}

Se desarrolló una tecnología de medición del calor específico de los aceites térmicos y de soya usados en la freidora de papas de la UEB Industrial Ceballos de Ciego de Ávila. Se determinó que el aceite térmico es muy estable en el tiempo y puede ser usado sin cambio durante más de 6 meses. El aceite de soya sufre un gran deterioro al estar en contacto con las papas y su calor específico crece muy rápido en el tiempo, por lo que se recomienda su sustitución a las 48 horas de uso, de lo contrario puede disminuir la eficiencia a la mitad de la que tenía cuando nuevo.

\section{Bibliografía:}

Fasina, OO. (2014). Predicting TemperatureDependence Viscosity and Specific Heat of Vegetable Oils from Fatty Acid Composition. Journal of the American Oil Chemist's Society 83(10),

Golato M. A , (2008). Propiedades térmicas de los aceites comestibles. Revista industrial y agrícola de Tucumán. 23, 34-42.

Marcano, R. (2012). Generadores de vapor. Fundamentos termodinámicos (Edit Michael J Moran, México).

Nwafor, OMI. (2003) The effect of elevated fuel inlet temperature on performance of diesel engine running on heat vegetable oil at constant speed conditions. Renewable Energy 28, 171-181, 